\title{
Refinement of laboratory animal welfare
}

\author{
Angelo Gazzano, Chiara Mariti \\ Department of Veterinary Science, \\ University of Pisa, Pisa, Italy
}

\begin{abstract}
In order to achieve an effective refinement of animal welfare, it is necessary to intervene on all phases of the experimental process: animal housing, experiment, rehoming of animals that have undergone experimentation, with an active intervention by researchers and veterinary surgeons.

It is crucial to know the ethology of the hosted animal species, in order to house animals in a physical and social environment that is as similar as possible to the environment in which that species live in natural conditions. A particular care must be devoted to experimental phase in which animal welfare is at greatest risk: a careful control by the veterinarian is necessary to identify the slightest signs of pain in the animal, intervening with an adequate analgesic therapy. At the end of the experimental phase, once the state of good health has been recovered, animals can be rehomed.
\end{abstract}

\section{Introduction}

In 1959, Russel and Burch in their book: "The principles of humane experimental technique", defined refinement as any activity addressed "to reduce the amount of stress imposed on those animals that are still used" in research. The authors indicated that improving experimental procedures involved avoiding cases of "direct inhumanity", avoiding cases of "contingent inhumanity", as well as improving the welfare during all the procedures accompanying the life of laboratory animals.

In 2005, a new definition of refinement was introduced by Buchanan-Smith et al.:1 "Any approach which avoids, minimizes the actual or potential pain, distress and adverse effects suffered at any time during the life of the animals involved, or which enhances their well-being as far as possible". What is particularly relevant in this definition is the reference to a positive state of welfare, well-being not corresponding to the absence of distress.

In order to effectively protect animal welfare, it is therefore necessary to intervene on all phases of the experimental process: animal housing, experiment, rehoming of animals that have undergone experimentation.

\section{Results \\ Housing}

It is crucial to know the ethology of the hosted animal species, in order to house animals in a physical and social environment that is as similar as possible to the environment in which that species live in natural conditions. Animals must also be used to be manipulated in a correct way by people. Early handling can have important positive effects, described in rodents ${ }^{2}$ and in domestic dogs. ${ }^{3}$

\section{Physical environment}

According to Fraser and Broom, ${ }_{4}$ who defined welfare as "the state of an animal as regards its attempts to cope with its environment", the physical aspects of the environment are very important and must allow the animal to be able to manage its relationships with it, for example hiding if the brightness is too intense or covering in the nest if the temperature perceived by the animal is too low.

In addition to controlling the temperature, brightness and air changes of the facility, it will be important to provide an adequate environmental enrichment of the cages, suitable for each individual species, in an adequate quantity avoiding disputes between the different individuals. Environmental enrichment is necessary to allow a correct development of the central nervous system, as many studies have shown in different animal species. 5 Furthermore, the cages should be wide enough to allow the most common behaviors in the specific development period: for example, it would be advisable to provide larger cages for young animals in order to allow social play.

Another aspect to pay attention to is the choice of the substrate covering the bottom of the rodent cage which, in addition to being comfortable and absorbent, must be in adequate quantity to allow natural behaviors such as digging, food search and creation of nests, for which it is necessary to provide suitable material.

It is necessary to remember that every displacement of the rodents from the old cage to the clean one causes stress, as the olfactory and pheromonal references disappear. It is therefore advisable to transfer a small amount of the previous substrate to the new cage to avoid disorientation of the animal that would find itself in an environment devoid of olfactory references.
Correspondence: Angelo Gazzano, Department of Veterinary Science, University of Pisa, Pisa, Italy.

Key words: Animal welfare; laboratory animals; refinement; 3R.

Conference presentation: this paper was presented at the Second Centro 3R Annual Meeting - 3Rs in Italian Universities, 2019, June 20-21, University of Genoa, Italy.

Received for publication: 28 October 2019. Accepted for publication: 6 November 2019.

This work is licensed under a Creative Commons Attribution NonCommercial 4.0 License (CC BY-NC 4.0).

\section{(c) Copyright: the Author(s), 2019}

Licensee PAGEPress, Italy

Biomedical Science and Engineering 2019; 3(s2):91 doi:10.4081/bse.2019.91

\section{Social environment}

To correctly structure the social environment, the knowledge of animal ethogram is essential. Some species (mouse) have solitary lifestyle habits and the life in a group, especially if made up of same sex individuals, is a source of stress and conflicts.

Other animals are, instead, gregarious and they need to live in groups of adequate size to permit the creation of stable social relationships. It is therefore necessary to form stable and compatible groups of animals, taking into consideration sex, age, reproductive status, familiarity between the subjects and previous experiences of group life. Moreover, it is also important that young animals can grow together with the mother avoiding early weaning.

\section{Experimental part}

It is the phase in which animal welfare is at greatest risk. First, it will be useful to habituate the animal to experimental procedures and equipment (for example habituating the rabbits to remain calm in the containment boxes to avoid injury to the spinal cord). In some animal species (dogs, nonhuman primates), the individual can be trained to collaborate with the researcher.

In this phase, a careful control by the veterinarian is necessary to identify the slightest signs of pain in the animal, intervening with an adequate analgesic therapy. For this purpose, the Grimace Scales, available for mouse, rat, rabbit and horse, can be very useful. Moreover, it is necessary to avoid that animals are subjected more than once to painful experimental tests. 


\section{Animal rehoming}

At the end of the experimental phase the animals that have "collaborated" with research will be housed in the facility for the time necessary to overcome the aftereffects of the experiment. Once the state of good health has been recovered, animals can be rehomed. In some cases, a behavioral rehabilitation program should be provided to make them adoptable. This is especially important for highly social animals such as dogs, whose behavior can also be altered by sensorial deprivation often experienced in the early stages of their development in research facilities.

\section{Conclusions}

The protection of laboratory animal welfare must be guaranteed in order to respect an ethical approach to research, and for its consequences on the scientific validity of experimental data. The refinement requires an active intervention by researchers and veterinary surgeons at all stages of the life of laboratory animals.

\section{References}

1. Buchanan-Smith HM, Rennie AE, Vitale A, et al. Harmonising the definition of refinement. Animal Welfare 2005;14:379-84.
2. Kosten T, Kim J, Lee H. Early life manipulations alter learning and memory in rats. Neurosci Biobehav Rev 2012;36:1985-2006.

3. Gazzano A, Mariti C, Notari L, et al. Effects of early gentling and early environment on emotional development of puppies. Appl Anim Behav Sci 2008; 110:294-304.

4. Fraser A, Broom D. Farm animals behaviour and welfare. London, UK: Bailliere Tindall; 1990.

5. Leger M, Paizanis E, Dzahini K, et al. Environmental enrichment duration differentially affects behavior and neuroplasticity in adult mice. Central Cortex 2015;25:4048-61. 